\title{
Digitale Mikroelektronik in Österreich
}

\author{
P. Rössler OVE, IEEE, A. Steininger IEEE
}

Online publiziert am 24. Juli 2015

(C) Springer Verlag Wien 2015

CrossMark

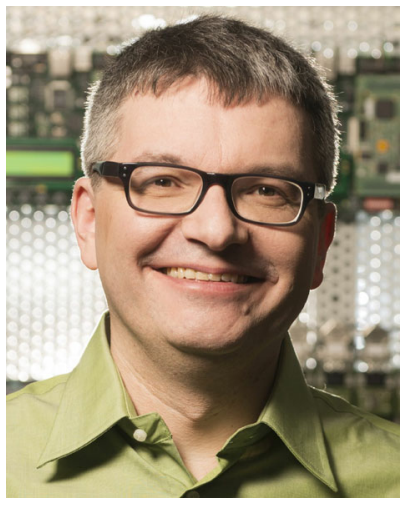

FH-Prof. Dipl.-Ing. Dr. techn. Peter Rössler

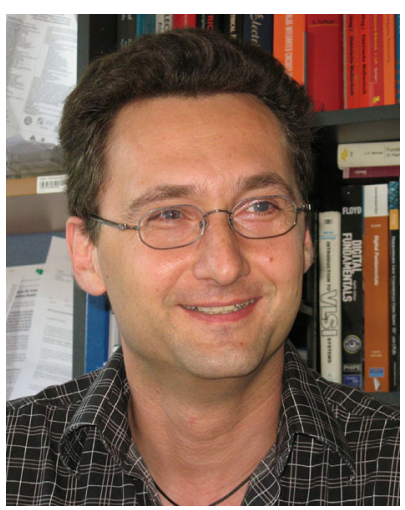

Ao. Univ.-Prof. Dipl.-Ing. Dr. techn. Andreas Steininger
Liebe Leserinnen und Leser,

die Mikroelektronik ist zweifellos die Schlüsseltechnologie unserer modernen Informationsgesellschaft. Viele alltägliche Dinge wie Smart Phones oder das Internet würden in ihrer heutigen Form ohne die Errungenschaften der Mikroelektronik schlichtweg nicht existieren. In der Automobiltechnik geht ein Großteil der Innovationen der letzten 20 bis 30 Jahre auf das Konto der Mikroelektronik, und auch in vielen anderen Applikationsbereichen wie der Medizintechnik, der Luftfahrt, der Industrieautomation oder der Konsumelektronik ist Mikroelektronik praktisch allgegenwärtig. Natürlich werden Forschung und (Produkt-)Entwicklung in diesem wichtigen Bereich auch von österreichischen Hochschulen und der in Österreich ansässigen Industrie betrieben. Nun gilt Österreich aber im Bereich der Hardwareentwicklung - und hier speziell auf Chip-Ebene - eher als Land der Analogtechnik, während man mit der Entwicklung von digitaler Mikroelektronik gemeinhin vor allem geografische Regionen wie Asien oder die USA assoziiert. Dennoch gibt es in Österreich auch im Bereich der digitalen Chips Aktivitäten und Produkte, die durchaus auf internationalem Niveau sind.

Im Rahmen dieses Schwerpunktheftes soll daher nun der Fokus auf digitale Mikroelektronik in Österreich sowie auf die in diesem Bereich tätigen Forschungsinstitutionen und Industriebetriebe gelegt werden. Die insgesamt acht Beiträge dieser e\&i-Ausgabe versuchen dazu, einen exemplarischen Überblick über die MikroelektronikSzene in Österreich zu geben. Bezüglich der Ausrichtung der Beiträge wurde im Sinne einer Collage aus Teilen unterschiedlichen Charakters bewusst auf detailliertere Vorgaben verzichtet, denn auch die Player und Aktivitäten sind höchst unterschiedlich. So finden sich in diesem Schwerpunktheft einerseits Übersichtsbeiträge und Surveys, aber auch Beiträge, die sich in sehr detaillierter Weise spezifischer Themen und Fragestellungen annehmen. Sowohl neue Technologien als auch Tools und Anwendungen in verschiedensten Applikationsbereichen werden behandelt.
Was sind nun die aktuellen Herausforderungen im modernen Digitaldesign? Hier ist an erster Stelle die ungeheure Integrationsdichte zu nennen, die ihrerseits wiederum durch extreme Miniaturisierung erkauft wird. Moderne Prozesstechnologien erlauben die Integration von Milliarden von Transistoren auf einem einzigen Chip und somit die Realisierung ganzer "Multi-Core Systems-on-Chip", d. h., auf dem Chip befinden sich gleich mehrere Rechenkerne samt Peripherie. Erschwerend kommt hinzu, dass die Abstraktion eines Transistors als Schalter in den nur mehr etwa $10 \mathrm{~nm}$ großen Strukturen längst an ihre Grenzen stößt; selbst Verbindungsleitungen bedürfen komplexer Modelle und weisen subtile Fehlermodi auf. Damit ergeben sich entsprechende Herausforderungen zur Beherrschung der Komplexität bei Entwicklung und in Folge auch Verifikation und Test derartig hochintegrierter Schaltungen.

Dieses Themas nimmt sich beispielsweise ein in diesem Heft enthaltener Beitrag an, der über einen von der FH Technikum Wien in Kooperation mit Infineon Technologies Austria entwickelten Verifikations-Ansatz berichtet, um die Brücke zwischen Pre-Silicon und Post-Silicon Verification von komplexen Mixed-Signal Systemon-Chip Designs zu schließen.

Ein bereits länger bekanntes Problem ist die stetige Zunahme der Leitungslängen am Chip - ein weiteres Resultat der Komplexität heutiger VLSI-Designs. Sie macht die Einhaltung der Zielvorgaben für das Timing immer schwieriger. Längst hat hier das WasserfallModell mit seiner strikten Trennung von Frontend- und BackendDesign ausgedient, und eine Logiksynthese ist ohne Kenntnisse über die physikalische Platzierung des Designs ineffizient. Bei komplexen VLSI-Chips müssen Timing-Aspekte zudem bereits beim Systemdesign, durch Anwendung von Paradigmen wie GALS (Globally Asynchronous, Locally Synchronous), berücksichtigt werden. In Zukunft werden in diesem Kontext zunehmend innovative Lösungen zur OnChip-Kommunikation gefragt sein, wie beispielsweise Network-onChip-Architekturen, die auch in einem hier enthalten Beitrag der TU Wien zur Sprache kommen. Bezüglich der heutzutage üblichen Taktund Signalfrequenzen sind, getrieben durch den Einsatz von HighSpeed-Schnittstellen wie GBit-Ethernet, USB 3.x oder SATA, auch Chip-extern Frequenzen von zig $\mathrm{GHz}$ nicht unüblich, womit man längst im HF-Bereich angelangt ist. Dies schafft, in Kombination mit dem hohen Pin-Count moderner VLSI-Chips (etwa sind bei aktuellen Hi-End FPGA-Bausteinen 1.000 Gehäusepins und mehr keine Seltenheit), wiederum entsprechende Aufwände bezüglich des Board Designs.

Eine weitere Folge der extremen Miniaturisierung der Transistorgeometrien in modernen Prozesstechnologien ist die zunehmende Anfälligkeit für Fehler, wie zum Beispiel durch ionisierende Partikel hervorgerufene "Single Event Upsets". Sie ist bedingt durch kleinere Spannungshübe, kleinere kritische Ladungsmengen, höhere Taktfre-

Rössler, Peter, Institut für Embedded Systems, FH Technikum Wien, Höchstädtplatz 6 1200 Wien, Österreich (E-Mail: peter.roessler@technikum-wien.at); Steininger, Andreas, Institut für Technische Informatik, Technische Universität Wien, Treitlstraße 1-3, 1040 Wien, Österreich (E-Mail: steininger@ecs.tuwien.ac.at) 
quenzen und letztlich auch durch die ungeheure Anzahl an Transistoren und Leitungen auf einem Chip. Hier gilt es, bereits im Entwurf gegenzusteuern. Diesem Thema widmen sich gleich zwei Beiträge im Schwerpunktheft: Der erste Beitrag, von drei Instituten der TU Wien, berichtet einerseits über die Entwicklung von verbesserten Simulationsmodellen für transiente Strahlungseffekte in Chips sowie weiters über die Entwicklung fehlertoleranter Routing-Algorithmen für die bereits erwähnten Networks-on-Chip. Im zweiten Beitrag wird ein neuartiges Tool zur Emulation von Single Event Upsets und anderer Fehler in FPGA-Bausteinen vorgestellt, das gemeinsam von Siemens Österreich und der FH Technikum Wien entwickelt wurde.

Die bereits mehrfach erwähnten FPGA (Field-Programmable Gate Array)-Bausteine haben seit etwas mehr als 20 Jahren den Bereich des digitalen Chip-Entwurfs geradezu revolutioniert; stellen diese doch eine im Vergleich zu Standardzellen-Chips unschlagbar kostengünstige Technologie für kleine und kleinste Stückzahlen dar und sind damit bevorzugte Plattform im Bereich Forschung, Lehre und Prototyping. Aber auch in Produkten werden FPGAs zunehmend eingesetzt, denn sie eröffnen gerade für kleinere Unternehmen einen niederschwelligen Zugang zum Markt. Im Beitrag von Joanneum Research wird am Beispiel eines Line-of-SightKommunikationssystems etwa beschrieben, wie FPGAs vorteilhaft zur Abarbeitung von performancekritischen DSP-Operationen verwendet werden können. Der Beitrag von Oregano Systems behandelt wiederum Details zur FPGA-Implementierung des Standards IEEE-1588, der zur hochgenauen Uhrensynchronisation in Computernetzwerken verwendet wird. Eine präzise Uhrensynchronisation ist auch notwenige Basis von Netzwerktechnologien, die nach dem Time-Triggered-Paradigma funktionieren. Zwei dieser Technologien, nämlich TTP und TTEthernet, werden samt Referenzen zu vorhandenen FPGA- und ASIC-Implementierungen im Beitrag der Firma TTTech beschrieben.

Auch in analogen Chips ist oftmals digitale Logik zur Parametrierung des Analogteils enthalten, beispielsweise, um Prozessschwankungen auszugleichen. Damit werden derartige Chips zu MixedSignal Designs mit all den bekannten Problemen. Einen Einblick in die Entwurfsmethodik der Digitalteile für derartige Mixed-Signal Designs gibt ein Beitrag des österreichischen Halbleiterherstellers ams.
Der pointiert geschriebene Übersichtsbeitrag liefert zudem auch einen informativen Überblick über die Entwicklung von Chips von ams für Audio- und Media-Player aus historischer Perspektive. Ein weiterer Beitrag der FH Kärnten beschreibt schließlich sehr detailliert den Entwurf von Multiratenfiltern zur Reduktion der Sample Rate von digitalen Signalen in Mixed-Signal Designs.

Es sei nochmals angemerkt, dass die Beiträge nur einen exemplarischen Überblick über die Aktivitäten in Österreich geben können. Neben den hier vorgestellten Institutionen findet man weitere bekannte österreichische Player im Bereich der Mikroelektronik etwa im Raum Graz (Firmen wie Dialog Semiconductors, Maxim, NXP sowie die TU Graz), in Linz (die Firmen DICE, Intel Mobile Communications Austria oder die Johannes Kepler Universität), im Wiener Umfeld (das Austrian Institute of Technology oder RUAG SPACE GmbH) sowie in Kärnten (z. B. die Firmen CISC Semiconductor und Lantiq sowie die Alpen-Adria-Universität Klagenfurt). Für eine umfassendere Information über Aktivitäten im Bereich der Entwicklung integrierter Schaltungen sei der Besuch der nationalen Tagung "Austrochip" empfohlen. Diese Tagung, die am 28. September 2015 zum nunmehr 23. Mal stattfand, hat sich im Laufe der Zeit zu einer bekannten Branchenplattform für Industrie und akademische Institutionen in Österreich entwickelt. Weiters sei das nationale „Microelectronic Systems Symposium" (MESS) genannt (vormals "Informationstagung Mikroelektronik"), das alle zwei Jahre in Wien stattfindet und Themenbereiche wie Sensorik, Nanoelektronik, Photonik und Embedded Systems behandelt. Schließlich fand von 14. bis 18. September 2015 auch die 41. „European Solid-State Circuits Conference” (ESSCIRC) in Graz statt. Auch der OVE Österreichischer Verband für Elektrotechnik hat sich im Jahr 2009 der steigenden Bedeutung der Mikroelektronik in Österreich mit der Gründung der "Gesellschaft für Mikroelektronische Systeme" (GMS) angenommen.

Wir dürfen uns abschließend noch bei allen Autoren der vorliegenden Beiträge bedanken. Ihnen, liebe Leserinnen und Leser, hoffen wir, mit diesem Schwerpunktheft einen informativen Überblick über die digitale Mikroelektronikszene in Österreich geben zu können und wünschen viel Vergnügen beim Lesen der Beiträge! 